\title{
Online Self-Calibration for Robotic Systems
}

\section{Doctoral Thesis}

Author(s):

Maye, Jérôme

Publication date:

2014

Permanent link:

https://doi.org/10.3929/ethz-a-010213941

Rights / license:

In Copyright - Non-Commercial Use Permitted 
DISS. ETH NO. 21912

\section{Online Self-Calibration for Robotic Systems}

A thesis submitted to attain the degree of Doctor of Sciences of ETH Zurich

(Dr. sc. ETH Zurich)

\section{Presented by \\ Jérôme Maye}

MSc en Informatique, Ecole Polytechnique Fédérale de Lausanne

Born November 13, 1981

Citizen of Chamoson (VS), Switzerland

Accepted on the recommendation of

Prof. Dr. Roland Y. Siegwart

Prof. Dr. Jan Peters

Dr. Paul Furgale

2014 


\begin{abstract}
Every robotic system has some set of parameters - scale factors, sensor locations, link lengths, etc.- that are needed for state estimation, planning, and control. Despite best efforts during construction, some parameters will change over the lifetime of a robot due to normal wear and tear. In the best case, incorrect parameter values degrade performance. In the worst case, they cause critical safety issues.

In this thesis, we are interested in developing automated systems that are capable of robust and long-term deployment in the hands of non-experts, so the automatic identification and update of these parameter values is highly important.

We present a generic algorithm for self calibration of robotic systems that utilizes two key innovations. First, it uses an information-theoretic measure to automatically identify and store novel measurement sequences. This keeps the computation tractable by discarding redundant information and allows the system to build a sparse but complete calibration dataset from data collected at different times. Second, as the full observability of the calibration parameters may not be guaranteed for an arbitrary measurement sequence, the algorithm detects and locks unobservable directions in parameter space using a combination of rank-revealing QR and singular value decompositions of the Fisher information matrix. The result is an algorithm that listens to an incoming sensor stream, builds a minimal set of data for estimating the calibration parameters, and updates parameters as they become observable, leaving the others locked at their initial guess.

We demonstrate our online self-calibration algorithm on three applications, including a laser rangefinder on a differential-drive robot, odometry sensors of a consumer vehicle, and digital cameras with various lenses. Each application is fully described and validated through an extensive set of simulated and realworld experiments.
\end{abstract}




\section{Résumé}

Tout système robotique a un certain nombre de paramètres-facteurs d'échelle, positions des capteurs, longueurs de liaison, etc.-qui sont nécessaires pour l'estimation de son état, la planification de ses tâches et son contrôle. Malgré tous les efforts entrepris lors de la construction, certains paramètres ont tendance à changer au cours de la durée de vie d'un robot en raison de l'usure normale. Dans le meilleur des cas, des valeurs incorrectes pour les paramètres dégradent les performances. Dans le pire des cas, elles peuvent provoquer des problèmes de sécurité critiques.

Dans cette thèse, nous nous intéressons au développement de systèmes automatisés qui peuvent être déployés de manière robuste et sur le long terme par des personnes non expérimentées. Dans cette optique, l'identification automatique et la mise à jour des ces valeurs relèvent d'une importance capitale.

Nous présentons un algorithme générique pour la calibration automatique de systèmes robotiques axé sur deux innovations majeures. Tout d'abord, il utilise une mesure basée sur la théorie de l'information, qui permet d'identifier et de stocker automatiquement de nouvelles séquences de données. Tout en réduisant la complexité computationelle en éliminant les données redondantes, cela permet de construire un jeu de données minimal pour la calibration à partir de données acquises à des moments différents. Deuxièmement, comme l'observabilité complète des paramètres de calibration ne peut être garantie pour une séquence arbitraire de données, l'algorithme détecte et bloque les directions non observables dans l'espace des paramètres en utilisant une combinaison de décomposition $\mathrm{QR}$ révèlant le rang et de décomposition en valeurs singulières de la matrice d'information de Fisher. Cela résulte en un algorithme qui traite un flux de données sensorielles, construit un jeu de données minimal pour estimer les paramètres de calibration, et met à jour ces paramètres au fur et à mesure qu'ils deviennent observables, tout en laissant les autres verrouillés à leur estimation initiale.

Nous démontrons la pertinence de notre algorithme incrémental de calibration automatique à l'aide de trois applications, comprenant un télémètre laser monté sur un robot de type differentiel, des capteurs d'odométrie d'une voiture de tourisme, et des appareils photo numériques avec différentes lentilles. Chaque application est présentée de manière détaillée et est validée par un ensemble de simulations et d'expériences avec des données réelles. 